\title{
INFLUENCE OF NEW TECHNOLOGIES IN JOURNALISM AND PROCESS OF PUBLIC OPINION: A NEW JOURNALISTIC CULTURE
}

\author{
Virginia Linares Rodríguez, Antonio Lucas Marín ${ }^{* *} \&$ Paloma Abejón \\ Mendoza***
}

The emergence of new technologies has brought a big impact in all society aspects but the most important, undoubtedly, has been the change in communication, since it connects people from any part of the world with some subject in common. This has been demonstrated, for example, in the Theory of Six Degrees of Separation: The Science of a Connected Age by Duncan J. Watts, sociology expert. Here he tries to confirm that we live "in a small world". The media is not a missing link in this technological chain, because it has known how to use all the resource that the network creates in order to spread its information. Today is not possible that a media doesn't have a space in a social network like Facebook or Twitter, being the last one the match of all media, because 140 letters is more than enough for an informative or opinion headline so thousands of followers can be informed in situ.

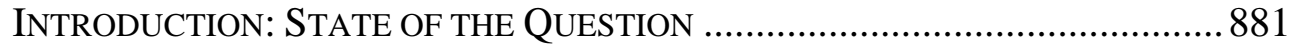

I. TRAditional Media FACING THE USE OF NEW TeChNOLOGIES...............883

A. Social Networks as Agents of Change ..................................... 887

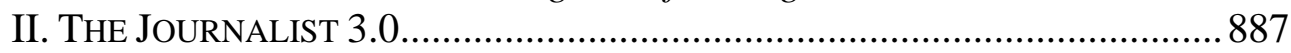

III. The INTERNET Audience: A New COMMUNICATIVE CulTuRE ............891

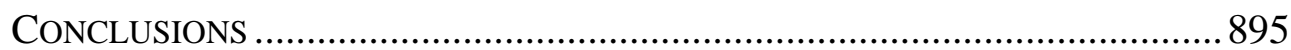

\section{INTRODUCTION: STATE OF THE QUESTION}

The evolution of Internet has been explosive since the launching of the first satellite, the Sputnik in 1957. From there it began to create the necessity of a transcendental change in the field of science and technology. This change has been excessive in all fields: The number of host connected, the domains, the traffic of information, the number of web pages, and currently the mass of social networks.

In 1969 it is made the first Internet host by establishing the connection

\footnotetext{
* Journalist, Ph.D. and Professor at the Complutense University of Madrid, Spain. Research fields: Journalism, Social Networks, Political Communication and New Technologies.

${ }^{* *}$ Full Professor of Sociology at Complutense University of Madrid, Spain. Research fields:

Sociology, Communication and Organizations.

${ }^{* * *}$ Professor Assistant and Doctor at Complutense University of Madrid. Research fields: Journalism, Social Networks and Political Communication.
} 
of four universities (UCLA, Stanford, Santa Bárbara and Utah) from there it begins the great network adventure ${ }^{1}$. This big expansion can be appreciated in the following Table 1 that shows the data available regarding the number of hosts, users and websites.

Table 1 Evolution of Internet in the World.

\begin{tabular}{llll}
\hline Year & Hosts & User & Websites \\
\hline 1985 & 1,961 & - & - \\
1990 & 313,000 & 4,000 & - \\
1992 & 992,000 & - & 50 \\
1995 & $6,642,000$ & $39,000,000$ & 25,000 \\
2000 & $93,047,000$ & $318,000,000$ & $1,000,000$ \\
2005 & $170,000,000$ & $1,081,000,000$ & $10,000,000$ \\
2010 & - & $1,858,000,000$ & $50,000,000$ \\
2015 & - & $3,585,700,000$ & $940,000,000$ \\
\hline
\end{tabular}

Source: Computer Industry Almanac Inc.

Hosts: Observatorio de la Sociedad de la Información.

Watching this data we can tell that the use of Internet has been growing very fast in the last 20 years, reaching a number of 3.585 billions of users in the world, an average of 39 users for every 100 individuals, a very significant number for the future of society information and society of new technologies.

In more definitely and precise numbers, Table 2, it's appreciate how United States keep its supremacy as leader until 10 years ago when it was overcome by China with the double.

Table 2 Countries with More Internet Users Connected (Number in Millions).

\begin{tabular}{lllll}
\hline Country & $\mathbf{2 0 1 5}$ & $\mathbf{2 0 1 0}$ & $\mathbf{2 0 0 5}$ & $\mathbf{2 0 0 0}$ \\
\hline China & 742.2 & 235.1 & 119.5 & 6.3 \\
United States & 312.3 & 234.2 & 197.8 & 110.8 \\
India & 243.0 & 108.4 & 50.6 & - \\
Brazil & 120.7 & 41.1 & 28.8 & 5.6 \\
Japan & 118.6 & 99.1 & 78.0 & 47.0 \\
Russia & 98.5 & 35.8 & 23.7 & - \\
Germany & 79.1 & 57.0 & 25.9 & 6.7 \\
Indonesia & 72.4 & 33.3 & 15.8 & 2.9 \\
Nigeria & 71.3 & 45.0 & 5.0 & 0.2 \\
Mexico & 62.4 & 25.4 & 16.9 & - \\
United Kingdom & 61.7 & 44.8 & 46.3 & 12.2 \\
France & 60.4 & 39.4 & 28.8 & 4.7 \\
Italy & 54.7 & 37.3 & 33.9 & 5.6 \\
Spain & 42.9 & 22.9 & 18.0 & - \\
Total World & $\mathbf{3 , 5 8 5 . 7}$ & $\mathbf{1 , 5 9 2 . 4}$ & $\mathbf{1 , 0 8 1}$ & $\mathbf{2 5 9 . 0}$ \\
\hline
\end{tabular}

Source: Computer Industry Almanac Inc. ITU

\footnotetext{
${ }^{1}$ A. LUCAS MARÍN, La nueva comunicación (Madrid: Trotta 2009).
} 
Sociology Expert Antonio Lucas Marín et al. in the book "La Nueva Comunicación", explain how the number of users of a country can give us an idea about its technological and economic power, because in order to know the much level of modernity they have we must use relevant data. In this sense, China is potentially dominant, although we have to mention that the United States level is almost equal to the European average.

In Spain also we can watch in Table 3, how increased exponentially the use of Internet in the last two decades. We past from $0.9 \%$ in 1997 to $73.6 \%$ in 2016, from 321 thousands users to almost 30 millions. Without doubt, a relevant information about how are using new technologies nowadays in the public opinion process. Its relevant has risen in social and political methods and at the same time it doesn't stop evolving. Besides, they are becoming a tool to motivate social change, giving its potential to boost transformation like public mobilization.

Table 3 Evolution of the Use of Internet (Extracted from Wave). $\%$ Individuals. *Individuals on thousands

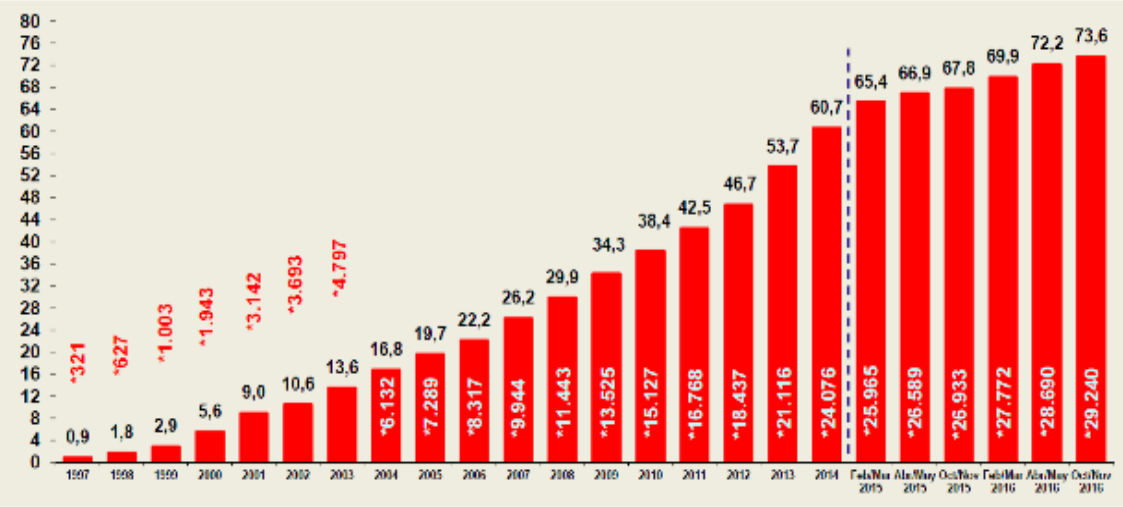

Source: AIMC - EGM.

New technologies make every citizen a sender, but the receiver can access the information by many methods, which create confusion in the system. The information we receive nowadays from testimonies of many users or from social media are not objective or transparent, but at least we, as receivers, have the possibility of knowing different points of views without forget the non-official characteristic of the source.

\section{TRaditional Media Facing the Use of NeW TeChNOLOGIES}

In our current world, which is constantly changing very rapid, is

\footnotetext{
${ }^{2}$ A. Lucas Marín \& V. Linares Rodríguez, Las nuevas tecnologías de la información y de la comunicación, in La nueva comunicación (A. Lucas Marín ed., Madrid: Trotta, 2009).
} 
special for the big transformation in human and social communication due the technological advances. Social Media gave new ways of communication, providing different patters of interaction and ways to express yourself, causing stimulation in citizens for bigger participations.

The capacity of spread the same message instantly and updated to a considerable number of people is no longer a surprise. However, if we consider human life in more primitive ages compared with our current life: Reading the newspaper, listening radio, watching TV or navigate in Internet, it represents an incredible change in human behavior ${ }^{3}$.

As DeFleur, Kearney and $\operatorname{Plax}^{4}$ pointed out, we must understand the mass media as the means of communication that transmits or sends messages to a wide and diverse audience; Therefore, we can say these media include television, radio, newspapers and, of course, Internet. Nowadays, there is no media of communication that does not have its space on the Internet.

In Table 4, we observe how almost all the mass media (newspapers, radio and television) from 1997 to 2016 have maintained, to a greater or lesser extent, their level of audience, with the exception of the Internet that had a really accelerated rise $(71.9 \%)$.

Table 4 Evolution of the Penetration of the Mass Media Spanish. $\%$ Individuals

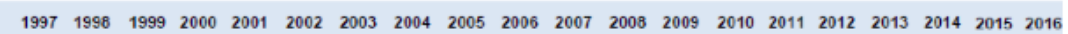
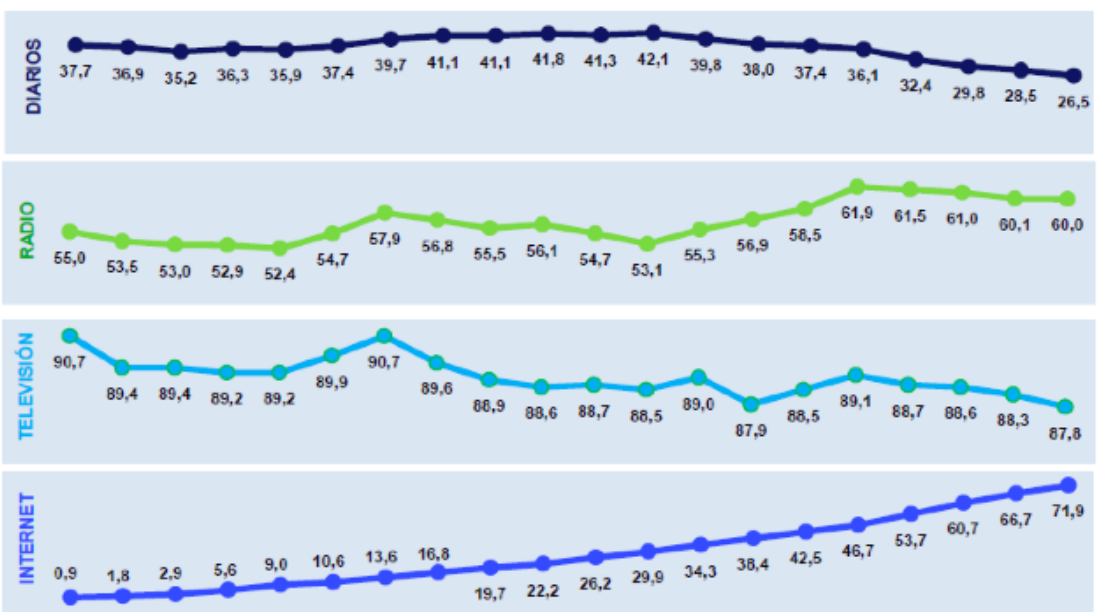

Source: EGM, 2016.

\footnotetext{
${ }^{3}$ A. Lucas Marín \& C. García Galera, Análisis de los medios de comunicación social, in La nueva comunicación 277 (A. Lucas Marín ed., Madrid: Trotta, 2009).

${ }^{4}$ M. L. DeFleur, P. Kearney \& T. G. Plax, Fundamentals of Human Communication, MounTAIN VIEW (CA.: Mayfield P. C 1993).
} 
According to Lucas Marín and García Galera ${ }^{5}$, these means have characteristics that cannot go unnoticed: 1) They require complex formal organizations for their operations, which requires trained staff, capital resources and financial control; 2) the media are directed towards large audiences, which cannot be specified; 3) mass communications are public, with their content open to all and relatively instructed and informal distribution; 4) the audience will be heterogeneous in its composition formed by people who will be in different cultures, living standards, education and sphere of power, etc.; 5) means can simultaneously establish with a large number of people, distant from the source and very separated from each other; 6) in mass communications, the relations between sender and audience are impersonal, so that an anonymous audience is directed by persons knowing only in their public role as communicators; 7) the media audience is an aggregate of individuals united by a common focus of interest, caught in an identical form of behavior and open to activating toward common ends.

Being more precise and according to the 3rd Annual Report prepared by $\mathrm{GAD}^{6}{ }^{6}$ in 2013 , a research and communication consultant, can be affirmed that the number of media followers through social networks is more than double the previous year, namely, the growth in the last twelve months was $128 \%$. Most of the media analyzed in this study are present in the three most widespread social networks in Spain (Facebook, Twitter and YouTube), with a total of more than twelve million and a half followers.

It can be seen, in Table 5, the number of followers in the generalist press networks almost tripled, with an increase of $184 \%$ in sporting newspapers up to $141 \%$ and in the media General information, exclusively digital grows $134 \%$. Meanwhile, more traditional media, despite its high penetration, continue to grow in followers: television at $107 \%$, conventional radio at $97 \%$ and radio at $67 \%$.

It is very important to note that the social network that most rose among traditional media users was Twitter, displacing Facebook, with more than 7 million followers in 2012 .

The television audience is much older than the Internet audience. This generational characteristic increases much more in social networks. Twitter is very distant from television because it has a young audience; users over

\footnotetext{
${ }^{5}$ A. Lucas Marín \& C. García Galera, Análisis de los medios de comunicación social, in La nueva comunicación 283 (A. Lucas Marín ed., Madrid: Trotta, 2009).

${ }^{6}$ The GAD3 study analyzed the presence of more than 50 media in Spain, classified in: 1) General press; 2) Economic press; 3) Sports press; 4) Free national newspapers; 5) Exclusively digital media; 7) Conventional radio strings; 8) Radio formula, 9) Digital TV and 10) digital channels.
} 
35 are not very present respecting the average Internet audience. These data reflect the need to build a series of very defined strategies on Twitter that are determined to a target audience.

Table 5 Mass Media in Social Networks.

\begin{tabular}{|c|c|c|c|c|c|c|c|c|c|c|c|}
\hline Prensa & & \multicolumn{4}{|c|}{ Facebook } & \multicolumn{3}{|c|}{ Twitter } & \multicolumn{3}{|c|}{ Youtube } \\
\hline & Medio & \multirow{2}{*}{\begin{tabular}{|l|} 
Icono \\
\end{tabular}} & \multirow{2}{*}{$\frac{\text { Fans }}{141.196}$} & Fotos & Videos & \multirow{2}{*}{\begin{tabular}{|l|} 
Icono \\
\end{tabular}} & \multirow{2}{*}{$\frac{\text { Seguidores }}{1.558 .366}$} & \multirow{2}{*}{$\frac{\text { Tweets }}{95.676}$} & \multirow{2}{*}{ Icono } & \multicolumn{2}{|c|}{ Suscriptores } \\
\hline & El Pais & & & 1.167 & 288 & & & & & $\beta$ & 1.954 \\
\hline & El Mundo & 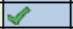 & 62.153 & 826 & 8 & $\sqrt{2}$ & \begin{tabular}{|c|}
739.769 \\
\end{tabular} & 19.080 & 26 & 8 & 0 \\
\hline \multirow{8}{*}{ Generalista } & $A B C$ & 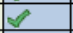 & 73.646 & \begin{tabular}{|l|l|} 
& 487 \\
\end{tabular} & $\Delta$ & 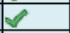 & $>138.757$ & 23.801 & 26 & 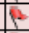 & 37 \\
\hline & \begin{tabular}{|l|} 
El Periódico \\
\end{tabular} & $\checkmark$ & 385.043 & 2223 & 0 & $\checkmark$ & 31.993 & 36.289 & 26 & 8 & 235 \\
\hline & La Gaceta & 23 & 42 & P & 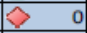 & $\checkmark$ & $>104.297$ & $>7.091$ & 26 & $\beta$ & 0 \\
\hline & \begin{tabular}{|l|} 
La Vanguardia \\
\end{tabular} & $\checkmark$ & 8.357 & 80 & $>$ & 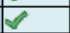 & $\begin{array}{r}95.230 \\
\end{array}$ & 68.511 & 28 & F & 543 \\
\hline & La Voz de Galicia & $\checkmark$ & > 29.689 & 561 & 3 & 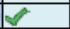 & 45.148 & 46.115 & 3 & P & 849 \\
\hline & La Razón & $\vartheta$ & 3.996 & 168 & $>$ & 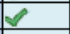 & 37.434 & 38.051 & 28 & 8 & 0 \\
\hline & Heraldo de Aragón & 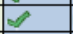 & 14.905 & \begin{tabular}{|l|} 
\\
\end{tabular} & 0 & $\infty$ & 18.768 & 21.877 & 2 & 6 & 12 \\
\hline & El Levante & 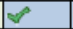 & 1) 7.737 & 35 & 0 & 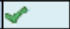 & 16.729 & 18.190 & 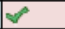 & $\beta$ & 29 \\
\hline \multirow{3}{*}{ Económicos } & Expansión & $\checkmark$ & 12.993 & D 310 & 0 & $\checkmark$ & 74.588 & $>27.583$ & 8 & $\beta$ & 49 \\
\hline & \begin{tabular}{|l|} 
El Economista \\
\end{tabular} & 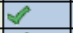 & 12.468 & D 224 & 8 & $\checkmark$ & 58.678 & 29.917 & 28 & $\beta$ & 50 \\
\hline & Cinco Días & 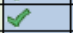 & $>10.464$ & 307 & $\Delta$ & $\checkmark$ & 43.710 & $\triangle 28.608$ & 26 & $\beta$ & 0 \\
\hline \multirow{3}{*}{ Deportivos } & Marca & 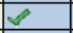 & 568.710 & 2.044 & 8 & $\checkmark$ & 753.453 & 73.730 & 8 & 8 & 6.789 \\
\hline & El Mundo Deportivo & $\checkmark$ & $>151.178$ & $\begin{array}{ll} & 209 \\
\end{array}$ & $>$ & $\checkmark$ & $\triangle 569.023$ & 45.689 & 26 & 6 & 20 \\
\hline & AS & 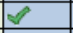 & $>263.343$ & 718 & 352 & $\checkmark$ & $>331.006$ & $>31.516$ & $\checkmark$ & $\beta$ & 1.438 \\
\hline Gratuitos & 20 Minutos & $\mathscr{V}$ & $>29.410$ & \begin{tabular}{|l|}
197 \\
\end{tabular} & 0 & $\checkmark$ & 147.117 & 25.866 & 28 & P & 55 \\
\hline Gratuitos & Qué! & 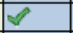 & 32.328 & 89 & 8 & $\checkmark$ & 8.175 & $>12.897$ & 2 & $\beta$ & 0 \\
\hline & Cadena Ser & $\checkmark$ & 61.183 & 30 & O & 26 & 128.559 & $\Delta 21.952$ & 2 & 8 & 197 \\
\hline & \begin{tabular}{|l|} 
Onda cero \\
\end{tabular} & $\vartheta$ & 80.816 & 97 & 3 & 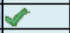 & 23.832 & $>\quad 6.952$ & 28 & B & 0 \\
\hline Radio Generalista & COPE & $\vartheta$ & $>22.230$ & 121 & 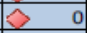 & 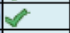 & 28.129 & 44.548 & 28 & $\beta$ & 1090 \\
\hline & RNE1 & ss & $\Leftrightarrow$ & D & 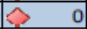 & 3 & 21.721 & $\Leftrightarrow \quad 2.596$ & 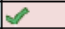 & 8 & 7 \\
\hline & ABC- Punto Radio & 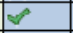 & $\hat{1} \quad 3.707$ & \begin{tabular}{|l|} 
\\
\end{tabular} & $\hat{\gamma}$ & $\checkmark$ & 9.809 & $\Leftrightarrow \quad 9.854$ & 2 & 6 & 0 \\
\hline & 40 Principales & $\vartheta$ & 449.115 & 63 & 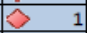 & 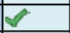 & 170.316 & 7.613 & 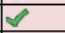 & p & 6.327 \\
\hline & \begin{tabular}{|l} 
Europa FM \\
\end{tabular} & $\checkmark$ & 457.269 & 765 & $\Delta$ & 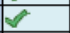 & $\triangle \quad 70.371$ & $\begin{array}{l}7.519 \\
\end{array}$ & 26 & P & 474 \\
\hline & Cadena 100 & 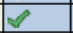 & 383.026 & 5.854 & $>$ & 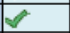 & 30.511 & $>2.502$ & $\checkmark$ & 8 & 2.374 \\
\hline Radio Formula & \begin{tabular}{|l|} 
Kiss FM \\
\end{tabular} & $\vartheta$ & $>174.117$ & \begin{tabular}{|l|}
513 \\
\end{tabular} & \begin{tabular}{|l|}
156 \\
\end{tabular} & 3 & 3.413 & 4.483 & 26 & P & 0 \\
\hline & \begin{tabular}{|l|} 
Cadena Dial \\
\end{tabular} & $\vartheta$ & $>73.331$ & 34 & 8 & 3 & 14.830 & 801 & 28 & 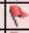 & 318 \\
\hline & M80 Radio & $\vartheta$ & $>65.183$ & 81 & 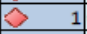 & $\checkmark$ & 3.939 & 3.428 & 28 & $\beta$ & 22 \\
\hline & Antena 3 & $\mathbb{V}$ & 561.474 & \begin{tabular}{|l|l|} 
& 973 \\
\end{tabular} & \begin{tabular}{|l|}
128 \\
\end{tabular} & $\checkmark$ & 267.494 & $\begin{array}{r}9.829 \\
\end{array}$ & $\because$ & $\beta$ & 96.973 \\
\hline & \begin{tabular}{|l} 
RTVE \\
\end{tabular} & 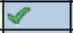 & 127.556 & \begin{tabular}{|l|} 
\\
\end{tabular} & 0 & 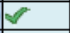 & 379.431 & 16.133 & $\because$ & 6 & 60.713 \\
\hline$T V$ & \begin{tabular}{|l|} 
Tele 5 \\
\end{tabular} & 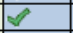 & 289.740 & $\begin{array}{r}990 \\
\end{array}$ & 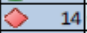 & $\vartheta$ & 152.772 & 14.588 & 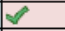 & $\beta$ & 76 \\
\hline & \begin{tabular}{|l|} 
Cuatro \\
\end{tabular} & 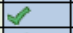 & 258.774 & \begin{tabular}{|l|l|} 
& 419 \\
\end{tabular} & 2 & $\checkmark$ & $>\quad 82.982$ & $>\quad 7.873$ & $\checkmark$ & 8 & 0 \\
\hline & La Sexta & $\theta$ & $>86.292$ & 1.188 & 0 & 36 & 138.896 & 88.992 & $\checkmark$ & $\beta$ & 100.028 \\
\hline & Público & 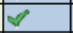 & 209.927 & $\begin{array}{ll}147 \\
\end{array}$ & D & $\checkmark$ & 221.650 & 60.719 & 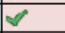 & $\beta$ & 0 \\
\hline & La Información & $\theta$ & 106.355 & \begin{tabular}{|l|}
103 \\
\end{tabular} & D & $\checkmark$ & 267.222 & 29.597 & $\checkmark$ & F & 1.135 \\
\hline & \begin{tabular}{|l|} 
Libertad Digital \\
\end{tabular} & $\vartheta$ & 30.678 & $\begin{array}{r}237 \\
\end{array}$ & $\hat{\theta}$ & $\checkmark$ & 15.080 & D 16.369 & 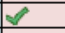 & P & 35.883 \\
\hline & \begin{tabular}{|l|} 
El Confidencial \\
\end{tabular} & 23 & 12.934 & 53 & 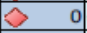 & $\checkmark$ & 72.514 & 28.540 & 26 & 6 & 7 \\
\hline Dialtales & \begin{tabular}{|l} 
Huffington Post \\
\end{tabular} & 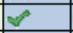 & 13.803 & 22 & 2 & $\checkmark$ & 28.159 & 542 & $\checkmark$ & $\beta$ & 1.281 \\
\hline Digitales & \begin{tabular}{|l|l|l|} 
El Confidencial Digital \\
\end{tabular} & $\theta$ & O & P & 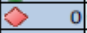 & $\checkmark$ & 34.123 & P 14.602 & 8 & $\beta$ & 1 \\
\hline & PR Noticias & 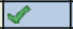 & D 4.817 & 146 & O & 2 & 28.273 & 27.429 & 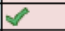 & P & 1.230 \\
\hline & El Plural & 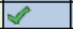 & 16.481 & 42 & 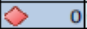 & 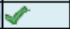 & 13.428 & D 3.823 & $\theta$ & $\beta$ & 0 \\
\hline & \begin{tabular}{|l|} 
Periodista Digital \\
\end{tabular} & $\vartheta$ & 6.795 & 12 & 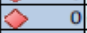 & $\checkmark$ & 11.759 & 15.657 & 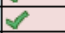 & F & 2.645 \\
\hline & El Imparcial & $\checkmark$ & 4.162 & 0 & 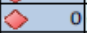 & $\checkmark$ & 6.386 & 3.766 & 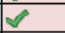 & $\beta$ & 0 \\
\hline & Cotizalia & 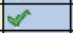 & 6.438 & 0 & 0 & $\checkmark$ & 9.845 & 23.127 & $\checkmark$ & 8 & 0 \\
\hline & \begin{tabular}{|l|} 
Libre Mercado \\
\end{tabular} & 23 & 3.808 & 86 & 0 & $\checkmark$ & 5.931 & P 6.014 & 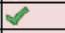 & $\beta$ & 701 \\
\hline & Invertia & $\checkmark$ & 5.608 & D & O & 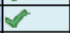 & 4.057 & 141.140 & 28 & p & 0 \\
\hline Digitales & Negocios & 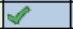 & 606 & P & D & 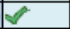 & 6.447 & 14.761 & $\theta$ & $\beta$ & 0 \\
\hline Económicos & Finanzas & 8 & O & P & D & $\checkmark$ & 5.253 & D 14.645 & 26 & 8 & 0 \\
\hline & \begin{tabular}{|l|} 
Economía Digital \\
\end{tabular} & $\checkmark$ & 1.083 & D & 0 & $N$ & 4.607 & 19.187 & $x$ & 3 & 0 \\
\hline & \begin{tabular}{|l|} 
Bolsamanía \\
\end{tabular} & $\vartheta$ & 1.211 & D & 0 & $\checkmark$ & 3.382 & 16.987 & 8 & $\beta$ & 126 \\
\hline & Infobolsa & $\infty$ & 1.540 & 0 & P & 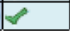 & 1.292 & 762 & 28 & 5 & 0 \\
\hline
\end{tabular}

Source: EGM, 2016.

The development of multimedia products has once again changed the scope regime of the viewer, in the sense that now they has access to a greater number of information, by the increase in the number of television channels available and through digital television. But also for the ability to 
connect through Internet broadcast channels both through the portals of the television networks and through the broadcast offered in social networks.

\section{A. Social Networks as Agents of Change}

For Lucas Marín and García Galera, the arrival of the information society implies the emergence of the new media of masses, as consequences of the technological advances that are happening, called the new technologies information, and have been the ones that created revolution in the world of communication ${ }^{7}$.

Hopper $^{8}$ points out that digital media is a catalyst for contemporary communication and constitute a transformation in human communication. Social networks have introduced new patterns of communication, diversified content, created new forms of expression, fostered freedom of expression and stimulated greater participation and collaboration. This allows citizens to have the opportunity to tell their perspectives, question social norms and produce changes in society. People can express themselves in many ways and participate freely in events, because social networks are less centralized and hierarchical, and are based on democratic structures. It is a new social form of communication that, although massive, is produced, received and perceived individually reaching the entire planet ${ }^{9}$. People have the power in their own hands to create ties with others persons and create, change, and share digital content with them ${ }^{10}$.

Social networks are characterized by many points of production and distribution. This is related to what is known as civil journalism, a new form of journalism that shows that social networks are available to the public, that is to say the media and the citizen can publish or access information on an equal basis ${ }^{11}$.

\section{THE JOURNALIST 3.0}

The current journalism is betting on the presence of citizen journalism

\footnotetext{
${ }^{7} \mathrm{Ibid}$, at 289.

${ }^{8}$ P. Hopper, Understanding Cultural Globalization [online] (Cambridge: Polity Press 2007), available on http://www.sciencedirect.com/science/article/pii/S0007681309001232 (last visited April 8, 2014).

${ }^{9}$ M. Castells, Communication Power (Oxford: Oxford University Press 2009).

${ }^{10}$ M. Kaplan \& J. Blakley, The Business and Culture of Social Media: In Search of the PeOPle Formerly KNown As the Audience (Barcelona: The Norman Lear Center 2009).

${ }^{11}$ M. Albertini Ríos, Análisis del discurso periodístico sobre el papel de las redes sociales en la Primavera Árabe (Trabajo Fin de Grado de Periodismo not published. Madrid: Universidad Complutense de Madrid 2014).
} 
through spaces enabled for it. Many of the online press releases have a space where readers can participate by posting opinions and how much information they want. It's a practical way of not denying an existing reality.

The journalist must do what he has done so far, but better, for this he must be prepared to work in multimedia, knowing how to write well and have basic concepts of image and sound management. The journalist cannot forget what matters most in the Information Society is the ability to interpret, make analysis, deliver explanations and make projections. The journalist must remember that his role is still telling stories that fascinate audiences and above all, it must be ethical.

The old passive audience is now active: Users can inform each other, globally and in local areas, making intensive use of the technological tools they have at their disposal, through the large distribution channel called Internet.

According to Manuel Moreno ${ }^{12}$, part of his work published in the Annual Report of the Journalistic Profession 2015 on "How to succeed in Networks: Journalists have never had so many tools to take advantage of our work, and among them there are social networks". These, according to him, are at this time a great source of information and a powerful tool to publish the contents of the press, under a medium and outside of it. And today, a journalist who sets up his own media is perfect to enter the market without incurring excessive marketing costs.

According to the VI Surge of the Social Networking Observatory of The Cocktail Analysis ${ }^{13}$ and the VI Annual Survey on Social Networks of the Internet Advertising Bureau ${ }^{14}$, nine out of ten people who use the Internet on a regular basis have at least one profile in a social network, and 99\% of Internet users know that they exist even if they do not use them. The use of networks is like any instrument of information, distribution, and, therefore, has its technique, conditioning and responsibilities, which also vary from one network to another. For journalists, the first decision is to choose a profile, whether it is itself or linked to a media of communication.

\footnotetext{
${ }^{12}$ M. MoReno, Cómo triunfar en las redes sociales (Barcelona: Gestión 2000, 2015);

Observatorio de Redes Sociales, VI OlEADA DEL OBSERVATORIO DE REDES SOCIALES DE THE COCKTAIL ANALYSIS [online], at 77 (2014), available on https://es.slideshare.net/TCAnalysis/6oleada-observatorio-redes-sociales (last visited February 20, 2017).

${ }^{13}$ Observatorio de Redes Sociales, VI Oleada del ObSERVATORIo de Redes Sociales de The COCKTAIL ANALYSIS [online] (2014), available on https://es.slideshare.net/TCAnalysis/6-oleadaobservatorio-redes-sociales (last visited February 20, 2017).

${ }^{14}$ IAB, VI EsTUdio ANUAL SOBRe REDES SOCIALES DE LA INTERNET ADVERTISING BUREAU [online] (2015), available on http://www.iabspain.net/wpcontent/uploads/downloads/2015/01/Estudio_Anual_Redes_Sociales_2015.pdf (last visited December 19, 2016).
} 
In the following Table 6 development and use of social networks, we can see how $49.2 \%$ of 2,423 professionals surveyed for the Annual Report of the $\mathrm{AMP}^{15}$ consider definitive that the development and use of social networks has boosted the image of the journalist, compared to $30.9 \%$ that believe is provisional.

Table 6 Development and Use of Social Networks.

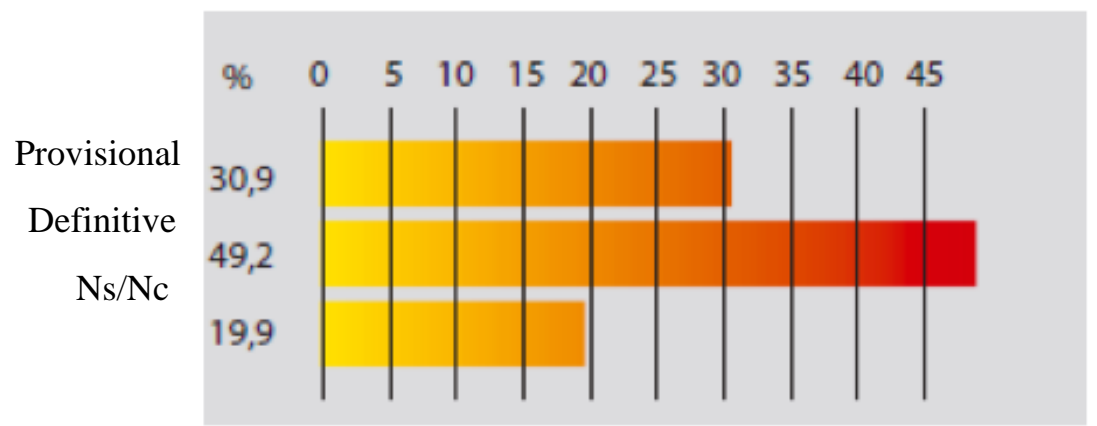

Source: Annual report of the AMP (2015).

We also observe in Table 7, which share opinions equally when, asked whether such presence should be a complement to their professional activity $(50.1 \%)$ or should be done on their own initiative and according to their own interests $(49.9 \%)$.

Table 7 The Presence of Journalists in Social Networks.

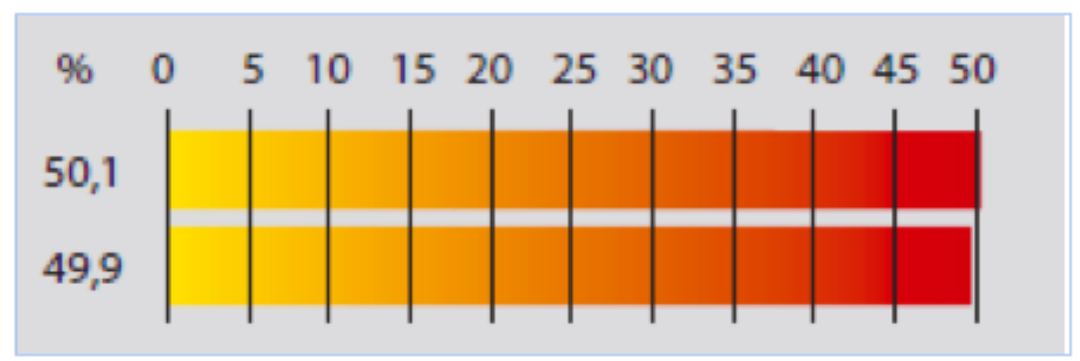

Source: Annual report of the AMP (2015).

In this area is the professional who must mark his own rules, his line of subjects and the tone of his contributions. In case the journalist appears to be linked with a medium, usually has to be subject to increasingly frequent norms of such media. It is usual, for example, that is not allowed to retweet or share the contents of competing media. A practice, according to Moreno,

${ }^{15}$ APM, Informe Anual de la Profesión Periodística 2015 (Madrid: APM 2015). 
that kills the potential of social media ${ }^{16}$. This is one of the reasons why citizens, involved in informational tasks (citizen journalists), have become the fifth power, the guardians of the fourth power.

Companies prefer journalists who has more than one ability, like being capable of producing interactive information, forum moderator, video editor, network expert, web 2.0 specialist, hypertext editor and user content integrator, publisher of online news streams, content manager, in short, journalist 3.0. In fact, $71 \%$ of those surveyed in the Annual Report of the APM 2015 believes that is necessary a training to do journalistic tasks with those of editor or content manager, as can be seen in Table 8 .

Table 8 Multitask Journalism.

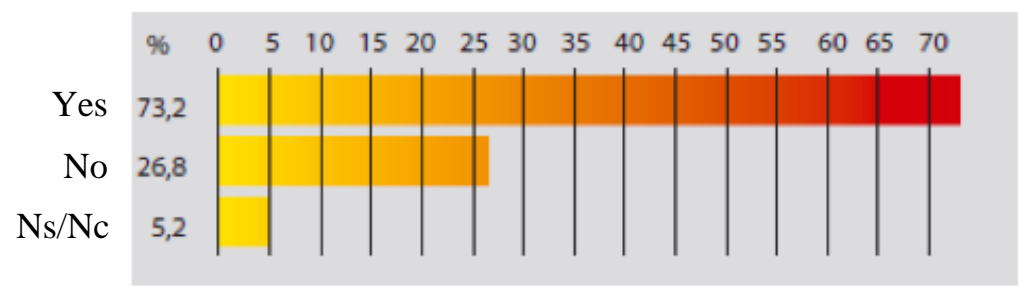

Source: Annual report of the AMP (2015).

It should be noted, in any case, the approval consumers give to the information they receive from the media does not lead them to dispense with these as the main source of information. This is shown in Table 9, where $64 \%$ of Spanish people prefer the information produced by journalists in the media, while the alternative where these professionals are not needed, such as social networks or blogs have a much lower predilection: $22 \%$. The same thing happens with press offices or institutional communication $(10 \%)$.

Table 9 Preference of Users for Information Sources.

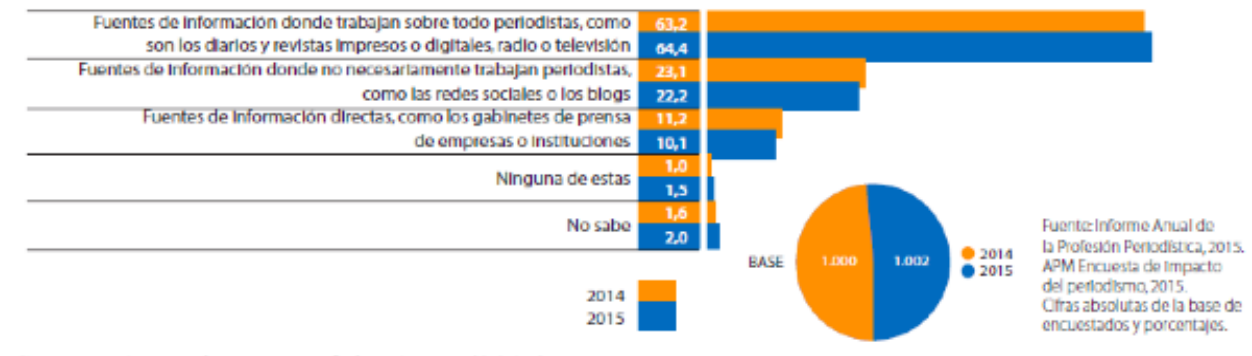

Source: Annual Report of the AMP (2015).

\footnotetext{
${ }^{16}$ M. Moreno, Cómo triunfar en las redes sociales. Barcelona: Gestión 2000. Observatorio de Redes Sociales (2014) VI Oleada del Observatorio de Redes Sociales de The Cocktail Analysis [online], 49 (2015), available on https://es.slideshare.net/TCAnalysis/6-oleada-observatorio-redes-sociales (last visited February 20, 2017).
} 
In the same way, Spanish people assign journalism an influence on the world of politics, economy, culture and sport that has even increased this year with respect to previous surveys (Table 10).

Table 10 Level of Influence of Journalists (\%).

\begin{tabular}{lllll}
\hline & Politics & Economy & Sport & Culture \\
\hline Much & 51.1 & 27.5 & 43.1 & 25.8 \\
Something & 23.0 & 27.5 & 25.9 & 31.5 \\
Little bit & 13.9 & 26.5 & 18.8 & 30.1 \\
Nothing & 9.7 & 15.7 & 8.5 & 10.0 \\
Ns/Nc & 2.4 & 2.7 & 3.8 & 2.4 \\
\hline
\end{tabular}

Source: Annual Report of the Journalistic Profession, 2015.

APM Journalism Impact Survey, 2015.

Concrete data of the respondent base and percentages.

On the other hand, the studies of Jung, Kim and Gil de Zúñiga ${ }^{17}$, say that the activation of so-called social capital through social networks results in changes in people's behavior.

Politics, society, and journalism, are facing multiple technological and information changes that are changing the way they present themselves to society. Along with the disruption of the monopoly of the figure of the sender, for example the way of campaigning is facing another great change: The multiplication of sources, which undoubtedly generates new processes of public opinion.

\section{The INTERNET AUdiEnCE: A New COMMUNICATIVE Culture}

Advances in new communication technologies have drastically changed the culture of information and communication not only in the sender, the channel and the message but also in the receiver; Therefore we cannot leave aside one of the most significant elements in the communicative process, the audience.

Based on Hiebert, Ungurait and Bohn ${ }^{18}$ and Lucas Marín ${ }^{19}$ the audience of any media must have at least five basic characteristics:

\footnotetext{
${ }^{17}$ N. Jung, Y. Kim \& H. Gil de Zúñiga, The Mediating Role of Knowledge and Efficacy in the Effects of Communication on Political Participation, MASS COMMUNICATION AND SOCIETY [online], (14) (2001), available on http://www.tandfonline.com/doi/full/10.1080/15205436.2010.496135?scroll=top\&needAccess=true (last visited April 20, 2014).

${ }^{18}$ R. E. Hiebert, D. F. Ungurait \& T. W. Bohn, Mass Media V. An Introduction to Modern COMMUNiCATION (New York: Longman 1988).

${ }^{19}$ A. Lucas Marín, Sociología de las organizaciones: Influencia de las tecnologías de la información y la comunicación (Madrid: Fragua, 2013).
} 
1. The audiences are formed by individuals who have certain skills that allow them to share certain experiences. These individuals consciously choose what they see in the media, since any member of the audience acts generally as an individual and not as mass, throughout the whole communicative process.

2. The audience is always quantitatively significant. However, the size of the audience is relative, since what can be a very large audience for a book may be inconsequential for a television program or almost nil for any event on any social network.

3. The audience is usually heterogeneous. The subjects that form part of it represent a wide variety of social categories. Some media seek specific receivers, with certain peculiarities, although this audience is made up of heterogeneous groups of people. This is called selective heterogeneity.

4. The audience is relatively anonymous. The communication takes place with the ignorance on the part of the sender of who is communicating. However, the audience studies allow knowing the professional, intellectual, personal, etc., characteristics of the receivers.

5 . The audience is physically separated from the issuer; But not only in space but also in time.

It should be noted that, when addressing the term "audience", we must take into account another series of classifications that are made of $\mathrm{it}^{20}$. For example, television audiences (those who have the TV on) are not the actual audiences (those who watch a particular program). Similar situations also occur with the rest of the media. In Table 11, we can clearly see the audience data of the Spanish media in 2015.

Table 11 Audience of the Mass Media Spanish, 2015.

\begin{tabular}{llll}
\hline Mass Media & $\begin{array}{l}\text { Total } \\
\text { (Thousands of people) }\end{array}$ & Males (\%) & Women (\%) \\
\hline Newpapers & 10,878 & 61.2 & 38.8 \\
Supplements & 3,937 & 43.5 & 56.5 \\
Magazines & 14,332 & 41.2 & 58.8 \\
Radio & 23,981 & 51.8 & 48.2 \\
TV & 35,067 & 48.6 & 51.4 \\
Exterior (advertising) & 29,070 & 50.4 & 49.6 \\
Cinema & 1,452 & 50.1 & 49.9 \\
Internet & 27,748 & 50.0 & 50.0 \\
\hline
\end{tabular}

Source: EGM, 2016.

\footnotetext{
${ }^{20}$ A. Lucas Marín \& C. García Galera, Análisis de los medios de comunicación social in La nueva comunicación, 179 (A. Lucas Marín ed., Madrid: Trotta, 2009).
} 
For their part, Straubhaar and LaRose point out that "a major transformation in the world of communication technology occurs when individual technologies begin to interact with others" ${ }^{21}$. In this respect García, García and Vinuesa ${ }^{22}$ inferred that the main star of new societies, without a doubt, is Internet, since it contributes to the better development of the communicative processes, the production and the handling of the information, being the most characteristic traits of a mediated audience. For this reason, Miége ${ }^{23}$ defined the Internet as an emblematic structure of the information society and the globalized world.

Meanwhile, for Castells ${ }^{24}$ Internet is the essential communication medium of the information age. For the first time in history, it allows instant communication from many to many. It has already deeply penetrated our way of working, informing, relating, learning and living. But what do we know about the effects of the Internet on society? Futurology, fantasy and ideology project a deformed image of what can be an Internet-based world. And yet, social research can bring materials to an objective knowledge of the real reach of the Internet. But in the end, the evolution of the Internet depends on its use by society, that is us. The challenge posed by the Internet is that it amplifies and enhances the human mind, both in its creative and destructive version. That is why knowledge of its uses and effects is essential to adapt the most powerful information, and communication technology to our values, interests and projects.

In the following Table 12, we can see the most visited Internet sites by the Spanish people in 2016.

Eltantawy and Wiest ${ }^{25}$ indicate that people as receivers or audiences, are the nucleus of communication for social change, whether is by dialogic or by digital. Communication is about individuals and, therefore, their role is to facilitate interaction between them. The participatory action of social media technologies has allowed new forms of communication to be mainstreamed for political and social change processes.

\footnotetext{
${ }^{21}$ J. StraubhaAR \& R. LaRose, Media Now: Communications Media in the Information Age 90 (Massachusetts: Cengage Learning, 1999).

${ }^{22}$ C. García, V. García \& L. Vinuesa, Las posibilidades de participación de los ciudadanos en campañas institucionales de comunicación mediante páginas web in Ética de la comunicación: problemas y recursos (M. Diezhandino \& Watt eds., Madrid: Editorial Foro Complutense, 2002). ${ }^{23}$ B. Miége, La pensée communicationnelle (París: Grenoble, 1995).

${ }^{24}$ M. CASTELls, LA GalaXia InTERNET (Barcelona: Plaza \& Janes Editores 2001).

${ }^{25}$ N. Eltantawy \& J. B. Wiest, Social Media in the Egyptian Revolution: Reconsidering Resource Mobilization Theory, (5) INTERNATIONAL JOURNAL OF COMMUNICATION [online] 1207-1224 (2011), available on http://ijoc.org/index.php/ijoc/article/view/1242/597 (last visited April 1, 2014).
} 
Table 12 Most Visited Internet Sites, 2016.

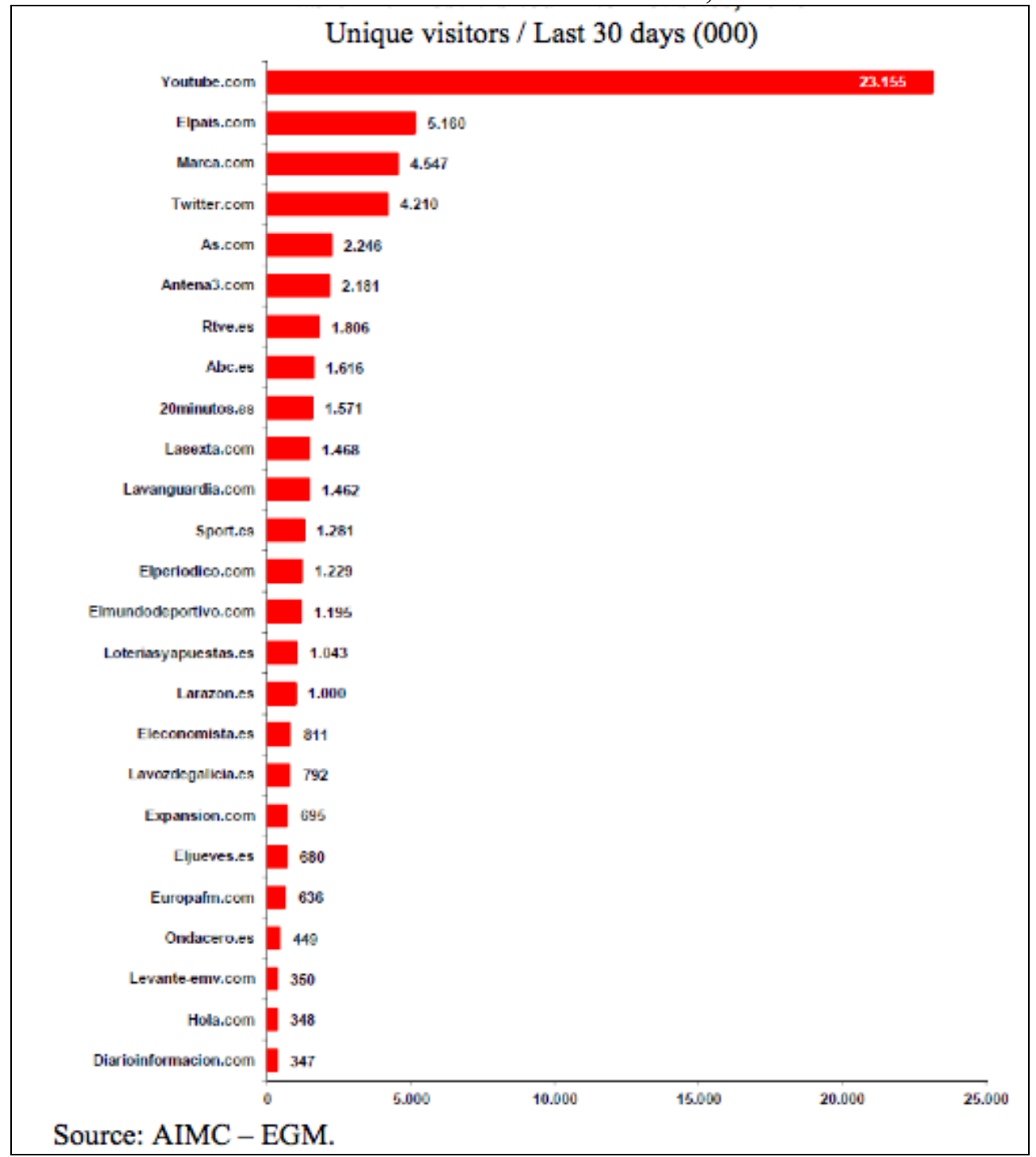

Albertini Ríos ${ }^{26}$ tells us, for example, in the Egyptian revolution, social networks were keys in promoting citizen journalism as a new way of talking about revolutionary events. They provided a platform in which minute-byminute citizens contributed with news stories, expressing themselves and talking about their own versions of reality by using new media in a more

\footnotetext{
${ }^{26}$ M. Albertini Ríos, Análisis del discurso periodístico sobre el papel de las redes sociales en la Primavera Árabe. Trabajo Fin de Grado de Periodismo not published (Madrid: Universidad Complutense de Madrid 2014).
} 
creative way, for example: uploading photos, videos or comments, as well as disseminating information to different media social networks offered "forums where they publish their own words and images to the outside world through regional and transnational media"27. Through them, "citizen journalists" tell their own stories and these patterns of political expression are crucial in the development of democratic discourses.

For this reason we can speak about the creation of a new communicative culture generated around the unique social spectators. It is relevant to mention the case of the relationship between television presenters and their viewers (as transmitters and receivers, respectively) through the networks ${ }^{28}$. The presenter of television becomes in that moment, a necessary intermediary to narrate the content in live, and in personal social networks, since they continue maintaining a connection of its image like presenter, and in some way demonstrating that still outside of its work continues to maintain its role as communicators, becoming the main influencer for the audience, as it can comment on what they are seeing or reading on social networks.

In Table 13, we can see how social viewers and the number of comments multiplies considerably on social networks, more and more people watch television with a second screen and comment on Twitter.

Table 13 Unique Social Users.
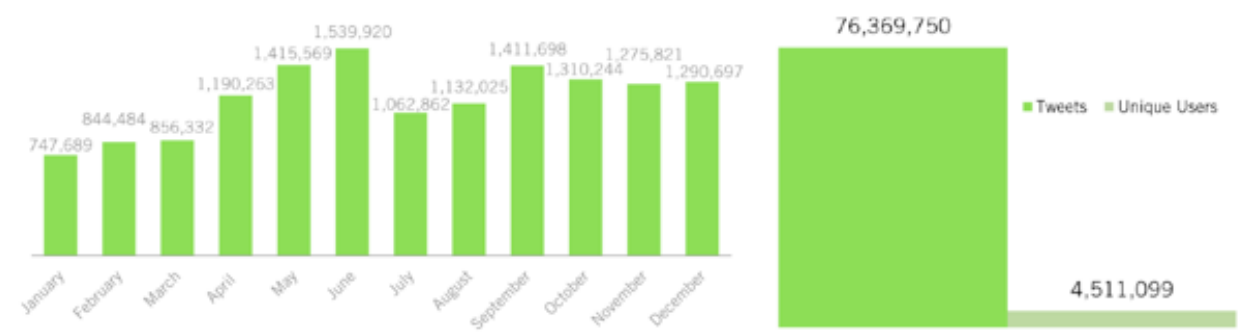

Source: Tuitele, 2013. La Sociedad de la Información en España.

\section{CONCLUSIONS}

Trying to understand the reality of the Network, the behavior of Internet users, and therefore understand the future that comes to us in this

\footnotetext{
${ }^{27}$ S. Khamis \& K. Vaughn, Cyberactivism in the Egyptian Revolution: How Civic Engagement and Citizen Journalism Tilted the Balance, (13) ARAB MEDIA AND SOCIETY [online] 1 (2011), available on http://arabmediasociety.sqgd.co.uk/articles/downloads/20110603105609_Khamis.pdf (last visited March 11, 2014).

${ }^{28}$ J. Sánchez Moreno, Análisis del discurso y la imagen periodística, Trabajo Fin de Grado de Periodismo not published (Madrid: UCM, 2014).
} 
dynamic and constant field, we can say that the Internet has a set of components that must be taken into account to understand its functioning. In the first place, the techno-meritocratic culture is manifested in the pursuit of its beginnings of scientific and academic excellence. Next, we must talk about virtual community culture. And finally mention the entrepreneurial culture, which we can see reflected in many of its greatest exponents, such as the founders of many companies: from Apple to Google or Facebook to a long etcetera.

The representation of the media in society plays a transcendental role in building a positive image of different elements through networks, Facebook or Twitter, as well as the definition of their identities and relationships. In addition, their perspectives dominate the articles in a way that frames the represented reality. In this way, it can be said that journalistic discourse is not only a reflection of reality, but a way of constructing reality that satisfies the needs of journalism and the media social representations tend to be the most prescriptive, imposing themselves on journalists and is observed in speeches.

However, the feeling of professional crisis persists because in recent years the environment in which journalists have developed their work has significantly changed and many things need to be redefined.

The variety of social networks allows the media to use the network to interact with their audience: TV channels have been positioned primarily on the YouTube online video platform, where they have a quarter million fixed subscribers, but where download hundreds of thousands of videos every day. The press has found on Twitter its strongest ally in the social network, where it has nearly five million regular followers, most of who are clustered in the general press and sports.

Therefore, and in light of the data exposed throughout the article we can deduce that the audience in the media through social networks grows exponentially. Respecting to studies from previous years, the growth experienced in the last twelve months has been $128 \%$. The growth of users in each medium must be added the proliferation of new digital information media that interact with their audiences through social networks.

Issuers have also detected a change in the way information is transmitted, and have experienced modifications in the way the audience interacts with them. Now you can watch information and comment at the same time, there is a continuous feedback. There has been a transformation in the communication process because the receivers are no longer passive. For all these reasons, we come to the conclusion that social networks have become a necessity for any journalist and media because they allow them to 
know all the opinions and reactions about what happens regarding their work at the same time of the diffusion, there is access to direct sources, and in addition they can be knowledgeable of the operation of a content through these new means.

In short, social networks, along with the new information and communication technologies, are an essential part of a complex and interrelated set of contextual variables of journalism and society, being a tool of great importance for both. 